\title{
Interleukin- 6 and interleukin-10 gene polymorphisms and the risk of further periodontal disease progression
}

\section{Georgios CHATZOPOULOS(a) Aikaterini-Ellisavet DOUFEXI(b) Larry WOLFF(a) \\ Anastasia KOUVATSI(c)}

(a) University of Minnesota, School of Dentistry, Department of Surgical and Developmental Sciences, Minneapolis, MN, USA.

(b) Private practice, Thessaloniki, Greece.

(c)Aristotle University of Thessaloniki, Faculty of Sciences, Department of Genetics, Development and Molecular Biology, Thessaloniki, Greece

Declaration of Interests: The authors certify that they have no commercial or associative interest that represents a conflict of interest in connection with the manuscript.

\section{Corresponding Author:}

Georgios S. Chatzopoulos

E-mail: chatz005@umn.edu.

https://doi.org/10.1590/1807-3107bor-2018.vol32.001 1

Submitted: August 22, 2017

Accepted for publication: December 12, 2017

Last revision: January 12, 2018

\begin{abstract}
Susceptible genotypes to periodontal disease are associated with disease onset and progression. The aim of this study was to examine the effect of gene polymorphisms on the risk of further disease progression and the need for further treatment among adults with chronic periodontal disease. Sixty-seven patients diagnosed with chronic periodontitis were grouped according to genotype status and risk of further progression of disease and tooth loss. All individuals were clinically evaluated for probing pocket depth, clinical attachment loss and bleeding on probing at baseline and 45 days after treatment. Blood samples were collected at baseline and genotyping of the polymorphisms in IL-6 (rs1800796) and IL-10 (rs1800872) genes were performed by PCR. Following DNA separation and genotyping, 65.7\% of the patients were homozygous carriers of the IL-6 -572G and 49.3\% were carriers of the IL-10 -592A allele. Individuals at risk of disease progression ranged from $7.5 \%$ to $62.7 \%$ based on the criteria used. Carriers of the IL-10 -592A allele were significantly associated with $\mathrm{BOP} \geq 30 \%$ and therefore exhibited a higher risk of further periodontal breakdown $(p=0.018)$ with an odds ratio of 1.18. None of the other definitions of disease progression were significantly associated with the examined IL- 6 and IL-10 genotypes ( $p>0.05$ ). IL-10 polymorphism was associated with an increased risk of further disease progression and the potential need for further treatment following non-surgical periodontal treatment. Susceptible IL-6 genotypes were not associated with the risk of persisting or recurrent disease activity.
\end{abstract}

Keywords: Interleukin-6; Interleukin-10; Disease Progression; Periodontitis.

\section{Introduction}

Periodontal disease is defined as a multifactorial inflammatory disease, which results from the interaction between periodontal pathogens and the host immune response. Microbial products initiate an inflammatory response leading to local tissue destruction including connective tissue and bone support loss, which may ultimately result in tooth loss. ${ }^{1}$ The virulence factors of the subgingival microbes disrupt the immune surveillance and elevate the virulence of the microbial community leading to a dysbiotic community and disruptive tissue homeostasis including periodontal ligament, cementum and 
alveolar bone. ${ }^{2}$ The high prevalence of periodontal disease, which affects nearly half of adults in the United States with $8.5 \%$ of these individuals having been diagnosed with severe periodontal disease. Individuals with periodontal disease incur the economic burden associated with the management of periodontitis and this further underscores the importance of implementing new therapeutic interventions. ${ }^{3}$ Approximately $10-15 \%$ of the population appears to progress rapidly from gingivitis to periodontitis and a few sites within individual patients or a small percentage $(8-12 \%)$ of patients within a given population were found to be unresponsive to routine periodontal treatment. ${ }^{4,5}$ The detection of these patients before the initial periodontal treatment is critical and may influence the clinicians treatment approach.

Initial periodontal therapy consists of oral hygiene instructions and non-surgical periodontal treatment aiming to improve a patient's quality of life as well as to preserve, improve and maintain longterm the natural dentition in healthy periodontal tissues. ${ }^{6}$ Risk of periodontal disease progression has been reported in the literature to be associated with smoking history, diabetes mellitus, age, poor oral hygiene, furcation involvement, residual pocket depths, frequency of supportive periodontal care and genetics. ${ }^{7-11}$ It is of paramount importance to identify periodontal disease progression as soon as possible in order to minimize the risk of irreversible periodontal bone loss. Today, the clinical diagnosis of chronic periodontal disease is based on clinical and radiographic findings of the periodontal tissues ${ }^{12}$, while this visual method may only detect the current status for the presence or absence of disease. Traditional clinical criteria are often insufficient for measuring the degree of susceptibility to future periodontal disease progression or determining the appropriate treatment plan for each individual. ${ }^{13}$

Identifying probable genetic polymorphisms associated with progression and onset of periodontal diseases may contribute in predicting periodontal treatment outcome and therefore may justify modifying the treatment plan accordingly. ${ }^{14}$ A number of different polymorphisms of various genes have been assessed in regards to the clinical outcomes after non-surgical periodontal therapy, but the lack of methodologically sound studies revealed the need for additional investigations. ${ }^{15}$ Individuals susceptible to periodontal disease progression following non-surgical periodontal therapy may benefit from adjunctive systemic antibiotics or other additional treatments. The use of genetic tests to predict the risk of future periodontal disease destruction may make it possible to individualize therapy (Personalized/Precision Medicine) with the intent of reducing the incidence of periodontally compromised teeth and tooth loss. The results from such a prognostic test may increase the accuracy of periodontal disease diagnosis as well as explaining unsuccessful treatments.

IL-6 has a significant effect in inflammation and is considered a pro-inflammatory cytokine that is secreted by immune cells, adipose tissues and muscles which regulates the immune reactivity and haemopoiesis. ${ }^{16}$ IL-6 has been associated with attachment loss and tissue destruction due to its effect on osteoclast differentiation. ${ }^{17,18,19}$ In addition, IL-6 is regulated at the transcriptional level and a polymorphism in the region of $-572 \mathrm{G} / \mathrm{C}$ plays a critical role in the regulation of transcription. ${ }^{20,21} \mathrm{On}$ the other hand, IL-10 is an anti-inflammatory cytokine that down-regulates the pro-inflammatory immune response of monocytes and macrophages. $.^{14} \mathrm{IL}-10$ is a differentiation factor that stimulates $B$ cells to secrete IgG, IgA and IgM which suppresses the pathological immune response driving the generation of CD4+ T cells. ${ }^{22}$ A significant increased risk of chronic periodontitis has been associated with IL-6 -572 G/C and IL-10 -592 C/A gene polymorphisms, especially in Caucasian Europeans. ${ }^{23,24}$

In periodontal therapy, it is of paramount importance to evaluate the response to active treatment that will detect potential persisting or recurrent disease activity and determine the need for additional therapy. The aim of this study was to examine the effect of interleukin-6 rs1800796 and interleukin-10 rs1800872 gene polymorphisms on the risk of further disease progression and the need for further treatment among adults with chronic periodontal disease. 


\section{Material and methods}

In this prospective cohort study, sixty-eight consecutive patients diagnosed with chronic periodontal disease were recruited from a private practice limited to Periodontics and Implant dentistry in Thessaloniki, Greece between September 2014 and June 2015. The purpose and the procedures of the study were completely analyzed, and all patients were given a written consent form in accordance with the Declaration of Helsinki. This research was approved by the Local Ethics Committee. The following inclusion criteria were considered for patient recruitment:

a. Diagnosis of chronic periodontitis with interproximal clinical attachment loss (CAL) $\geq 3 \mathrm{~mm}$, probing pocket depth (PPD) $\geq 5 \mathrm{~mm}$ and bleeding on probing (BOP) in two or more non-adjacent sites. ${ }^{25}$

b. Good general systemic health.

c. Age at initial appointment between 30 and 70 years.

d. Presence of at least 16 teeth in the oral cavity.

e. Ability to consent.

On the contrary, individuals were excluded from the study for one or more of the following conditions:

a. History of periodontal therapy (non-surgical and surgical) within the past 12 months.

b. History of severe medical disorders.

c. History of systemic diseases.

d. Need for antibiotic prophylaxis.

e. History of systemic antibiotic therapy in the past 3 months.

f. Pregnant or lactating females.

\section{Clinical examination}

Clinical measurements were performed by a single, calibrated (weighted kappa index of intra-examiner agreement $=0.88$ ) examiner (A.E.D) who was blinded to the genotype status of each patient. The periodontal examination included probing pocket depth (PPD), clinical attachment loss (CAL) and bleeding on probing (BOP) which were recorded utilizing a standard manual periodontal probe (15 UNC probe, Hu-Friedy, Chicago, IL, USA) at six sites around each tooth (mesio- buccal, mid-buccal, disto-buccal, mesio-lingual, mid-lingual, disto-lingual) for the whole mouth, except the third molars. Periodontal measurements were performed at baseline and at 45 days after non-surgical periodontal therapy.

\section{Periodontal therapy}

Active periodontal therapy included oral hygiene instructions and non-surgical supra- and sub-gingival debridement provided by a single, calibrated, experienced and blinded periodontist (A.E.D). Manual curettes (Hu-Friedy, Chicago, USA) and ultrasonic instrumentation (KaVo SONOsoft LUX, Kavo, Germany) were utilized. Patients were re-evaluated 45 days following the completion of active non-surgical periodontal treatment and the same clinical procedures as performed at baseline were carried out which included PPD, CAL and BOP by the same calibrated clinical examiner. The risk of progression of periodontal disease was determined on a site-level based on the presence of at least one site with residual PPD $\geq 6 \mathrm{~mm}$ ( $1^{\text {st }}$ definition) or $\geq 9$ sites with residual PPD $\geq 5 \mathrm{~mm}$ ( $2^{\text {nd }}$ definition $)$ at the re-evaluation of periodontal therapy (45 days). ${ }^{26}$ At the patient level, the risk of disease progression was assessed when full-mouth bleeding score was $\geq 30 \%$ ( $3^{\text {rd }}$ definition) after active non-surgical periodontal therapy. ${ }^{26}$

\section{Blood samples and genotyping}

The included individuals were considered susceptible or non-susceptible to chronic periodontitis, according to the IL-6 -572 G/C and IL-10 -592 C/A polymorphisms. Genomic DNA was extracted from drops of blood using a commercially available genomic DNA isolation kit (QIAamp, DNA mini blood kit, QIAGEN, Germany) according to the manufacturer's instructions. Genotyping of the polymorphisms in IL-6 -572 G/C (rs1800796) and IL-10 -592 C/A (rs1800872) genes was performed by polymerase chain reaction, in a final volume of $25 \mathrm{ul}$ according to the manufacturer's standard protocol. ${ }^{27}$ The genotype of random samples was confirmed by sequencing analysis.

The IL-6 polymorphism genotypes were determined by using the 5'-GGAGACGCCTTGAAGTAACTGC-3' 
and 5'- GAGTTTCCTCTGACTCCATCGCAG-3' primers to generate a PCR product of $163 \mathrm{bp}$ that was then digested with BsrBI (NEB) restriction enzyme. ${ }^{27}$ The $-572 \mathrm{C}$ allele lacks the digestion site, giving a fragment of $163 \mathrm{bp}$, while the $-572 \mathrm{G}$ allele gives two fragments of 102 and $61 \mathrm{bp}$. Primer pair 5'-CAACTTCTTCCACCCCATCTTT-3' and 5'-GTGGGCTAAATATCCTCAAAGTT-3' was used to amplify a 477 bp sequence containing the IL-10 polymorphism. ${ }^{27}$ Restriction enzyme RsaI (NEB) cleaved the $-592 \mathrm{C}$ specific PCR product into four fragments of 311, 116, 42 and $8 \mathrm{bp}$ and the $-592 \mathrm{~A}$ product into five fragments of 240, 116, 71, 42 and $8 \mathrm{bp} .{ }^{27}$ For the IL-6 and IL-10 polymorphisms the digested products were separated on polyacrylamide gels stained with silver nitrate. Patients carrying the genotype IL-6 GG (susceptible allele G) and/ or IL-10 CA or IL-10 AA (susceptible allele A) were classified as susceptible to chronic periodontitis, while the non-susceptible group were carriers of the non-susceptible genotype IL-10 CC and/or IL-6 GC or CC.

\section{Statistical analysis}

Descriptive analysis of the collected data was performed. Continuous variables are presented as mean $\pm S D$ and categorical variables are shown in frequency numbers and percentages. The chi-square test was utilized to determine the significance of a susceptible genotype regarding the risk of further disease progression. The odds ratios with their 95\% confidence intervals and corresponding $\mathrm{p}$-values for the samples were analyzed. All statistical procedures were performed with statistical software (SPSS v.19.0 for Windows, IBM, Armonk, USA). Statistical significance was set at $p \leq 0.05$. Calculation of the sample size was described in our previous report. ${ }^{27}$ The power of the analysis was performed to calculate sample size based on a pilot sample collection of five susceptible and five non-susceptible patients. Sample size was determined in order to achieve an $80 \%$ statistical power and $5 \%$ level of significance resulting in a minimum size of 34 per group (susceptible and non-susceptible).

\section{Results}

A total of 68 Caucasian patients with chronic periodontal disease were divided into two groups according to the presence or absence of the susceptible IL-6 and IL-10 genotype. One patient was excluded from the study because he failed to attend the follow-up appointment due to change of residency. Ultimately, the final population consisted of 67 Caucasians. The demographic characteristics, genotype and periodontal status of the included population are shown in Table 1. The total subject population included $32(47.8 \%)$ males and 35 (52.2\%) females with a mean age of $47.63 \pm 9.66$ years. A history of smoking was reported by 28 (41.8\%) of the included population, while 39 individuals (58.2\%) denied that they had ever been smokers. In regards to genotype status, $44(65.7 \%)$ individuals were homozygous carriers of the IL-6 -572G and were classified as susceptible to periodontal disease, while $22(32.8 \%)$ and $1(1.5 \%)$ were heterozygous and homozygous carriers, respectively of the IL-6 -572C genotype and were classified as non-susceptible. Thirty-three (49.2\%) patients were found to be susceptible to periodontal disease because they carried the susceptible genotype IL-10 -592 CA or AA, whereas $34(50.8 \%)$ were non-susceptible and carriers of the IL-10 -592 CC. The mean number of missing teeth along with the mean clinical measures of subjects in this population group may also be seen in Table 1.

Individuals at risk of disease progression included $62.7 \%$ of subjects based on the $1^{\text {nd }}$ definition (at least one site with residual PPD $\geq 6 \mathrm{~mm}$ ) and $31.3 \%$ based on the $2^{\text {nd }}$ definition $(\geq 9$ sites with residual PPD $\geq 5$ $\mathrm{mm}$ ), while at the patient-level, $7.5 \%$ of the included population were found to be at risk of further periodontal destruction (BOP $\geq 30 \%$ ). Distribution of IL-6 -572 G/C (rs1800796) and IL-10 -592 C/A (rs1800872) genotypes as well as clinical periodontal parameters at baseline and the $45^{\text {th }}$ day following periodontal treatment among individuals at risk or not at risk of periodontal disease progression as determined by the presence of at least one site with residual PPD $\geq 6 \mathrm{~mm}$ are shown in Table 2 . No significant differences $(p>0.05$ ) were found 
Table 1. Demographic characteristics, genotype and periodontal status of the included population.

\begin{tabular}{|c|c|}
\hline Characteristics & Total $(n=67)$ \\
\hline \multicolumn{2}{|l|}{ Gender (\%) } \\
\hline Males & $32(47.8)$ \\
\hline Females & $35(52.2)$ \\
\hline Age $($ mean $\pm S D)$ & $47.63 \pm 9.66$ \\
\hline \multicolumn{2}{|l|}{ Smoking (\%) } \\
\hline Yes & $28(41.8)$ \\
\hline No & $39(58.2)$ \\
\hline \multicolumn{2}{|l|}{ IL-6 -572 G/C (rs1800796) } \\
\hline \multicolumn{2}{|l|}{ Genotypes (\%) } \\
\hline GG & $44(65.7)$ \\
\hline GC & $22(32.8)$ \\
\hline $\mathrm{CC}$ & $1(1.5)$ \\
\hline \multicolumn{2}{|l|}{ Susceptibility (\%) } \\
\hline SCP & $44(65.7)$ \\
\hline NSCP & $23(34.3)$ \\
\hline \multicolumn{2}{|l|}{ IL-10 -592 C/A (rs1800872) } \\
\hline \multicolumn{2}{|l|}{ Genotypes (\%) } \\
\hline $\mathrm{CC}$ & $34(50.8)$ \\
\hline CA & $26(38.8)$ \\
\hline $\mathrm{AA}$ & $7(10.4)$ \\
\hline \multicolumn{2}{|l|}{ Susceptibility (\%) } \\
\hline SCP & $33(49.2)$ \\
\hline NSCP & $34(50.8)$ \\
\hline Missing teeth (mean $\pm \mathrm{SD}$ ) & $2.27 \pm 2.65$ \\
\hline $\mathrm{BOP}($ mean $\pm \mathrm{SD})$ & $84.23 \pm 29.71$ \\
\hline $\mathrm{PPD}($ mean $\pm \mathrm{SD})$ & $3.60 \pm 0.68$ \\
\hline $\mathrm{CAL}($ mean $\pm \mathrm{SD})$ & $3.79 \pm 0.75$ \\
\hline Sites with PPD $=4-6 \mathrm{~mm}($ mean $\pm \mathrm{SD})$ & $30.15 \pm 15.56$ \\
\hline Sites with PPD $\geq 7$ mm (mean $\pm S D)$ & $5.50 \pm 6.16$ \\
\hline Sites with $C A L=4-6 \mathrm{~mm}($ mean $\pm \mathrm{SD})$ & $35.7 \pm 16.60$ \\
\hline Sites with $C A L \geq 7$ mm (mean $\pm S D)$ & $6.86 \pm 7.27$ \\
\hline
\end{tabular}

IL: Interleukin; SCP: susceptible; NSCP: non-susceptible; BOP: bleeding on probing; PPD: probing pocket depth; CAL: clinical attachment level; SD: standard deviation.

between the IL-6 -572 G/C and IL-10 -592 C/A polymorphisms with the risk of disease progression. Clinical periodontal parameters were significantly different $(p<0.05)$ between the at risk and not at risk individuals at baseline and the $45^{\text {th }}$ day after the treatment.
Distribution of the examined genotypes and the clinical periodontal parameters among individuals at risk or not at risk of periodontal disease progression as determined by the presence of at least 9 sites with residual PPD $\geq 5 \mathrm{~mm}$ are demonstrated in Table 3. IL-6 -572 G/C (rs1800796) and IL-10 -592 C/A (rs1800872) polymorphisms were not associated with an increased risk of further disease progression $(p>$ $0.05)$, while all examined periodontal clinical measures were significantly different between the groups ( $p$ $<0.05$ ) both at baseline and the $45^{\text {th }}$ day following non-surgical periodontal therapy.

In Table 4, the distribution of IL-6 -572 G/C and IL-10 -592 C/A genotypes and the clinical parameters are shown among patients at risk or not at risk of disease recurrence as determined by the full-mouth bleeding score. The statistical analysis revealed that IL-6 rs1800796 gene polymorphisms are not associated with an increased risk of further disease progression following non-surgical periodontal therapy, while carriers of the IL-10 -592A allele were significantly associated with $\mathrm{BOP} \geq 30 \%$ and therefore exhibited a higher risk of further periodontal breakdown $(p=0.02)$ with an odds ratio of 1.18 (1.02-1.36). In particular, all included homozygous carriers of the IL-10 -592C and non-susceptible individuals $(\mathrm{n}=34,100 \%)$ showed no risk of further disease progression $(p=0.02)$. Individuals at risk of disease recurrence exhibited significantly $(p<0.05)$ higher PPD, CAL, BOP and sites with PPD and CAL between $4-6 \mathrm{~mm}$ as well as PPD and CAL $\geq 7 \mathrm{~mm}$ at 45 days following treatment when compared to those not at risk.

When smoking history and gender were considered as potential factors that may affect an individuals' risk of persisting or recurrent disease activity following active periodontal treatment, neither of them showed significant association ( $p$ $>0.05$ ). Both females and males as well as smokers and non-smokers of the included population demonstrated similar risk of disease progression after evaluating all three definitions (presence of at least one site with residual PPD $\geq 6 \mathrm{~mm}$ or presence of $\geq 9$ sites with residual PPD $\geq 5 \mathrm{~mm}$ or full-mouth bleeding score $\geq 30 \%$ ). 
Table 2. Distribution of IL-6 -572 G/C (rs 1800796) and IL-10 -592 C/A (rs1800872) genotypes as well as clinical periodontal parameters at baseline and the $45^{\text {th }}$ day following periodontal treatment among individuals at risk or not at risk of periodontal disease progression as determined by the presence of at least one site with residual PPD $\geq 6 \mathrm{~mm}$.

\begin{tabular}{|c|c|c|c|}
\hline \multirow{2}{*}{ Variable } & At risk & No risk & \multirow{2}{*}{ p-value* } \\
\hline & $(n=42,62.7 \%$ & $(n=25,37.3 \%$ & \\
\hline \multicolumn{4}{|c|}{ IL-6 -572 G/C (rs1800796) } \\
\hline \multicolumn{4}{|c|}{ Genotypes (\%) } \\
\hline GG & $29(65.9)$ & $15(34.1)$ & 0.49 \\
\hline GC & $12(54.5)$ & $10(45.5)$ & \\
\hline $\mathrm{CC}$ & $1(100)$ & $0(0)$ & \\
\hline \multicolumn{4}{|c|}{ Susceptibility (\%) } \\
\hline SCP & $29(65.9)$ & $15(34.1)$ & 0.45 \\
\hline NSCP & $13(56.5)$ & $10(43.5)$ & \\
\hline
\end{tabular}

IL-10 -592 C/A (rs1800872)

Genotypes (\%)

$\begin{array}{lcr}\text { CC } & 20(58.8) & 14(41.2) \\ \text { CA } & 17(65.4) & 9(34.6) \\ \text { AA } & 5(71.4) & 2(28.6)\end{array}$

Susceptibility (\%)

SCP

NSCP
11 (33.3)

$14(41.2)$
0.51

$20(58.8)$
0.84

(28.6)

Clinical periodontal parameters (baseline)

\begin{tabular}{|c|c|c|c|}
\hline $\mathrm{BOP}($ mean $\pm \mathrm{SD})$ & $85.37 \pm 29.00$ & $82.31 \pm 31.40$ & 0.69 \\
\hline PPD & $3.77 \pm 0.66$ & $3.31 \pm 0.62$ & 0.01 \\
\hline CAL & $3.99 \pm 0.70$ & $3.45 \pm 0.73$ & 0.004 \\
\hline Sites with PPD $=4-6 \mathrm{~mm}($ mean $\pm \mathrm{SD})$ & $32.93 \pm 15.30$ & $25.49 \pm 15.17$ & 0.06 \\
\hline Sites with PPD $\geq 7$ mm (mean $\pm S D$ ) & $7.12 \pm 6.51$ & $2.80 \pm 4.45$ & 0.01 \\
\hline Sites with $C A L=4-6 \mathrm{~mm}($ mean $\pm \mathrm{SD})$ & $38.45 \pm 15.56$ & $29.38 \pm 17.03$ & 0.03 \\
\hline Sites with $\mathrm{CAL} \geq 7 \mathrm{~mm}$ (mean $\pm \mathrm{SD}$ ) & $8.80 \pm 7.46$ & $3.61 \pm 5.70$ & 0.004 \\
\hline \multicolumn{4}{|l|}{ inical periodontal parameters (45 days) } \\
\hline $\mathrm{BOP}($ mean $\pm \mathrm{SD})$ & $15.71 \pm 13.68$ & $8.55 \pm 8.28$ & 0.21 \\
\hline $\mathrm{PPD}($ mean $\pm \mathrm{SD})$ & $2.94 \pm 0.48$ & $2.38 \pm 0.36$ & $<0.001$ \\
\hline $\mathrm{CAL}($ mean $\pm \mathrm{SD})$ & $3.24 \pm 0.57$ & $2.54 \pm 0.48$ & $<0.001$ \\
\hline Sites with PPD $=4-6 \mathrm{~mm}$ (mean $\pm \mathrm{SD})$ & $15.74 \pm 10.53$ & $5.93 \pm 6.69$ & $<0.001$ \\
\hline Sites with PPD $\geq 7$ mm (mean \pm SD) & $1.47 \pm 2.99$ & $0.00 \pm 0.00$ & 0.02 \\
\hline Sites with $C A L=4-6 \mathrm{~mm}($ mean $\pm \mathrm{SD})$ & $24.87 \pm 11.95$ & $10.73 \pm 9.97$ & $<0.001$ \\
\hline Sites with CAL $\geq 7 \mathrm{~mm}$ (mean $\pm \mathrm{SD}$ ) & $2.84 \pm 4.53$ & $0.81 \pm 1.51$ & 0.04 \\
\hline
\end{tabular}

IL: Interleukin; SCP: susceptible; NSCP: non-susceptible; BOP: bleeding on probing; PPD: probing pocket depth; CAL: clinical attachment level;SD: standard deviation. Footnote: IL-6 -572 G/C (rs 1800796) SCP genotypes were considered patients carriers of the GG genotype and IL-6 -572 G/C (rs 1800796) NSCP genotypes patients carriers of the C allele (GC+CC). IL-10 -592 C/A (rs 1800872) SCP genotypes were considered patients carriers of the A allele (AA+CA) and IL-10 -592 C/A (rs 1800872) NSCP genotypes carriers of the CC genotype. *Bold denotes statistical significance at $p<0.05$. 
Table 3. Distribution of IL-6 -572 G/C (rs 1800796) and IL-10 -592 C/A (rs1800872) genotypes as well as clinical periodontal parameters at baseline and the $45^{\text {th }}$ day following periodontal treatment among individuals at risk or not at risk of periodontal disease progression as determined by the presence of at least 9 sites with residual PPD $\geq 5 \mathrm{~mm}$.

\begin{tabular}{|c|c|c|c|}
\hline \multirow{2}{*}{ Variable } & At risk & No risk & \multirow{2}{*}{$p$-value* } \\
\hline & $(n=21,31.3 \%)$ & $(n=46,68.7 \%)$ & \\
\hline \multicolumn{4}{|c|}{ IL-6 -572 G/C (rs 1800796) } \\
\hline \multicolumn{4}{|c|}{ Genotypes (\%) } \\
\hline GG & $15(34.1)$ & $29(65.9)$ & 0.21 \\
\hline GC & $5(22.7)$ & $17(77.3)$ & \\
\hline $\mathrm{CC}$ & $1(100.0)$ & $0(0)$ & \\
\hline \multicolumn{4}{|c|}{ Susceptibility (\%) } \\
\hline SCP & $15(34.1)$ & $29(65.9)$ & 0.50 \\
\hline NSCP & $6(26.1)$ & $17(73.9)$ & \\
\hline
\end{tabular}

IL-10 -592 C/A (rs 1800872)

Genotypes (\%)

$\begin{array}{lll}\text { CC } & 10(29.4) & 24(70.6) \\ \text { CA } & 8(30.8) & 18(69.2) \\ \text { AA } & 3(42.9) & 4(57.1)\end{array}$

Susceptibility (\%)

$\begin{array}{lll}\text { SCP } & 11(33.3) & 22(66.7) \\ \text { NSCP } & 10(29.4) & 24(70.6)\end{array}$

Clinical periodontal parameters (baseline)

\begin{tabular}{|c|c|c|c|}
\hline $\mathrm{BOP}($ mean $\pm \mathrm{SD})$ & $96.43 \pm 16.37$ & $78.66 \pm 32.80$ & 0.02 \\
\hline PPD & $4.24 \pm 0.53$ & $3.30 \pm 0.52$ & $<0.001$ \\
\hline CAL & $4.44 \pm 0.57$ & $3.49 \pm 0.63$ & $<0.001$ \\
\hline Sites with PPD $=4-6 \mathrm{~mm}$ (mean $\pm \mathrm{SD})$ & $41.45 \pm 10.88$ & $25.00 \pm 14.69$ & $<0.001$ \\
\hline Sites with PPD $\geq 7$ mm (mean $\pm S D$ ) & $10.51 \pm 7.57$ & $3.22 \pm 3.62$ & $<0.001$ \\
\hline Sites with $C A L=4-6 \mathrm{~mm}($ mean $\pm S D)$ & $45.50 \pm 12.41$ & $30.31 \pm 16.17$ & $<0.001$ \\
\hline Sites with CAL $\geq 7 \mathrm{~mm}$ (mean $\pm \mathrm{SD}$ ) & $12.76 \pm 8.56$ & $4.17 \pm 4.61$ & $<0.001$ \\
\hline \multicolumn{4}{|l|}{ inical periodontal parameters ( 45 days) } \\
\hline $\mathrm{BOP}($ mean $\pm \mathrm{SD})$ & $20.70 \pm 16.91$ & $9.54 \pm 7.61$ & $<0.001$ \\
\hline $\mathrm{PPD}($ mean $\pm \mathrm{SD})$ & $3.24 \pm 0.47$ & $2.50 \pm 0.34$ & $<0.001$ \\
\hline $\mathrm{CAL}($ mean $\pm \mathrm{SD})$ & $3.56 \pm 0.55$ & $2.71 \pm 0.47$ & $<0.001$ \\
\hline Sites with PPD $=4-6 \mathrm{~mm}$ (mean $\pm \mathrm{SD})$ & $22.42 \pm 10.41$ & $7.36 \pm 6.11$ & $<0.001$ \\
\hline Sites with PPD $\geq 7$ mm (mean $\pm S D)$ & $2.50 \pm 3.97$ & $0.20 \pm 0.46$ & $<0.001$ \\
\hline Sites with $C A L=4-6 \mathrm{~mm}($ mean $\pm \mathrm{SD})$ & $31.08 \pm 10.81$ & $14.35 \pm 10.54$ & $<0.001$ \\
\hline Sites with $C A L \geq 7$ mm (mean $\pm S D)$ & $4.40 \pm 5.86$ & $1.02 \pm 1.57$ & 0.001 \\
\hline
\end{tabular}

IL: Interleukin; SCP: susceptible; NSCP: non-susceptible; BOP: bleeding on probing; PPD: probing pocket depth; CAL: clinical attachment level; SD: standard deviation.

IL-6 -572 G/C (rs 1800796) SCP genotypes were considered patients carriers of the GG genotype and IL-6 -572 G/C (rs 1800796) NSCP genotypes patients carriers of the C allele (GC+CC). IL-10 -592 C/A (rs 1800872) SCP genotypes were considered patients carriers of the A allele (AA+CA) and IL-10 -592 C/A (rs 1800872) NSCP genotypes carriers of the CC genotype. *Bold denotes statistical significance at $p<0.05$. 
Table 4. Distribution of IL-6 -572 G/C (rs 1800796) and IL-10 -592 C/A (rs 1800872) genotypes as well as clinical periodontal parameters at baseline and the $45^{\text {th }}$ day following periodontal treatment among individuals at risk or not at risk of periodontal disease progression as determined by the full-mouth bleeding score.

\begin{tabular}{lccc}
\hline \multirow{2}{*}{ Variable } & At risk & No risk & \\
\cline { 2 - 3 } & $n=5,7.5 \%$ & $n=62,92.5 \%$ & $p$-value* \\
\hline
\end{tabular}

IL-6 -572 G/C (rs1800796)

Genotypes (\%)

$\begin{array}{lcr}\text { GG } & 4(9.1) & 40(90.9) \\ \text { GC } & 1(4.5) & 21(95.5) \\ \text { CC } & 0(0) & 1(100)\end{array}$

Susceptibility (\%)

$\begin{array}{lll}\text { SCP } & 4(9.1) & 40(90.9) \\ \text { NSCP } & 1(4.3) & 22(95.7)\end{array}$

IL-10 -592 C/A (rs 1800872)

Genotypes (\%)

$\mathrm{CC}$

CA

AA

Susceptibility (\%)

SCP

NSCP

$5(15.2)$

$0(0)$

$28(84.8)$

0.02

Clinical periodontal parameters (baseline)

$\mathrm{BOP}$ (mean $\pm \mathrm{SD}$ )

PPD

$\mathrm{CAL}$

Sites with PPD $=4-6 \mathrm{~mm}$ (mean $\pm \mathrm{SD}$ )

Sites with PPD $\geq 7 \mathrm{~mm}$ (mean \pm SD)

Sites with $\mathrm{CAL}=4-6 \mathrm{~mm}($ mean $\pm \mathrm{SD})$

Sites with $\mathrm{CAL} \geq 7 \mathrm{~mm}$ (mean $\pm \mathrm{SD})$

Clinical periodontal parameters (45 days)

$$
\begin{aligned}
& \mathrm{BOP}(\text { mean } \pm \mathrm{SD}) \\
& \mathrm{PPD}(\text { mean } \pm \mathrm{SD}) \\
& \mathrm{CAL}(\text { mean } \pm \mathrm{SD}) \\
& \text { Sites with } \mathrm{PPD}=4-6 \mathrm{~mm} \text { (mean } \pm \mathrm{SD}) \\
& \text { Sites with } \mathrm{PPD} \geq 7 \mathrm{~mm} \text { (mean } \pm \mathrm{SD}) \\
& \text { Sites with } \mathrm{CAL}=4-6 \mathrm{~mm} \text { (mean } \pm \mathrm{SD}) \\
& \text { Sites with } \mathrm{CAL} \geq 7 \mathrm{~mm} \text { (mean } \pm \mathrm{SD})
\end{aligned}
$$

$$
100.0
$$

$100.00 \pm 0.00$

$4.63 \pm 0.66$

$4.80 \pm 0.75$

$40.10 \pm 4.77$

34 (100)

0.02

$3(11.5)$

$23(88.5)$

$5(71.4)$

$2(28.6)$

$34(100)$

0.77 


\section{Discussion}

This study investigated the role of IL-6 and IL-10 gene polymorphisms on the risk of disease progression following active non-surgical periodontal therapy in patients diagnosed with chronic periodontal disease. Sixty-seven consecutive patients of a private practice limited to Periodontics and Implant Dentistry received periodontal treatment and were followed-up 45 days after the periodontal therapy. Patient carriers of the IL-6 GG and IL-10 CA or IL-10 AA genotype were considered susceptible to periodontal disease. ${ }^{23,24}$ The hypothesis of the present investigation was that individuals susceptible to periodontal disease would demonstrate an increased risk of periodontal disease progression and persisting or recurrent disease activity following treatment as has been determined by previously accepted and validated criteria. ${ }^{26}$ We believe this is the first study that investigated this hypothesis. The results of our study suggest that IL-10 -592 C/A polymorphism is associated with a higher risk of periodontal disease progression following non-surgical periodontal therapy in a Caucasian population.

Although, in general, several long-term studies have demonstrated that patients with chronic periodontal disease respond favorably to non-surgical treatment, a number of these studies reported that a few sites within individual patients responded poorly leading to tooth loss and edentulism. ${ }^{4,5}$ Recognizing these patients is of paramount importance to prevent further periodontal destruction. Unfavorable response to conventional treatment may be attributed to poor plaque control, systemic conditions that impair healing, smoking, genetic factors and others. ${ }^{28}$ Research on the genetic background of periodontitis as well as other complex diseases has increased our current understanding and suggests that multiple genes and interactions between environmental and genetic factors may lead to susceptibility to periodontal disease. However, the current knowledge surrounding the genetic background of periodontal disease is still incomplete. ${ }^{29}$ Polymorphisms in the regulatory regions of genes may alter the expression of cytokines revealing an important role of genetic predictors of disease susceptibility and clinical measures..$^{30}$ More specifically, both IL-6 -572 G/C and IL-10 -592 C/A polymorphisms have been associated with an increased protein expression and inflammatory response. ${ }^{23,31}$

In the present study, we utilized drops of blood from patients with chronic periodontitis. Genotyping of the polymorphisms in IL-6 (rs1800796) and IL-10 (rs1800872) genes were performed using the real-time polymerase chain reaction technology. The included individuals exhibited $30.15 \%$ sites with PPD $=4-6 \mathrm{~mm}$ and more than a third of the sites showed CAL $=4-6 \mathrm{~mm}$. The mean number of sites with PPD $\geq 7 \mathrm{~mm}$ and $\mathrm{CAL} \geq 7$ was 5.50 and 6.86 , respectively. The clinical periodontal parameters of the baseline and the $45^{\text {th }}$ day after periodontal treatment of the "at risk" groups when compared to "no risk" groups as defined by the three different examined definitions (presence of at least one site with residual PPD $\geq 6 \mathrm{~mm}$ or presence of $\geq 9$ sites with residual PPD $\geq 5 \mathrm{~mm}$ or full-mouth bleeding score $\geq 30 \%$ ) were statistically significantly different $(p<0.05)$. Therefore, individuals "at risk" of further periodontal disease progression demonstrated a more severe disease status than the "no risk" group of patients. This finding may also confirm the examined criteria of increased risk of periodontal disease progression and persisting or recurrent disease activity following treatment. To the best of our knowledge this is the first study that aimed to investigate the potential association between gene polymorphisms and risk of periodontal disease progression. Therefore, our findings could not be compared to previous studies' results.

The present investigation demonstrated that carriers of the IL-10 -592A allele were significantly associated with BOP $\geq 30 \%$ and therefore showed a higher risk of further periodontal breakdown following non-surgical periodontal treatment. It has been shown that gingival inflammation plays a key role in the progression of periodontal diseases. ${ }^{5}$ In a study that included well-maintained, well-educated male individuals, periodontal pockets increased in number and in depth at areas with gingival inflammation. ${ }^{32}$ Gingival sites with bleeding on probing throughout a 26-year observation period demonstrated approximately $70 \%$ more attachment loss than non-inflamed gingival sites, yielding an odds ratio of 3.22 for the inflamed sites to result in 
attachment level loss. ${ }^{32}$ In another study that evaluated the use of bleeding on probing as an indicator for periodontal disease progression or disease stability, the majority of patients who experienced disease progression demonstrated a mean BOP score of greater than or equal to $30 \% .{ }^{33}$ On the other hand, individuals with BOP score less than or equal to $20 \%$ exhibited a significantly lower risk for further attachment loss. Moreover, a retrospective study reported that patients with $16 \%$ or more BOP sites were more likely to experience further periodontal breakdown during the maintenance phase of periodontal therapy. ${ }^{34}$

Future studies in different populations, with larger sample sizes and a longer follow-up period are required to examine various genetic polymorphisms of different genes. Bioinformatics may provide valuable information for patients with periodontal

\section{References}

1. Offenbacher S. Periodontal diseases: pathogenesis. Ann Periodontol. 1996 Nov; (1):821-78. https://doi.org/10.1902/annals.1996.1.1.821

2. Hajishengallis G, Lamont RJ. Beyond the red complex and into more complexity: the polymicrobial synergy and dysbiosis (PSD) model of periodontal disease etiology. Mol Oral Microbiol. 2012 Dec;27(6):409-19. https://doi.org/10.1111/j.2041-1014.2012.00663.x

3. Eke PI, Dye BA, Wei L, Thornton-Evans GO, Genco RJ. Prevalence of periodontitis in adults in the United States: 2009 and 2010. J Dent Res. 2012 Oct;91(10):914-20. https://doi.org/10.1177/0022034512457373

4. Johnson NW, Griffiths GS, Wilton JM, Maiden MF, Curtis MA, Gillett IR et al. Detection of high-risk groups and individuals for periodontal diseases. Evidence for the existence of high-risk groups and individuals and approaches to their detection. J Clin Periodontol. 1988 May;15(5):276-82. https://doi.org/10.1111/j.1600-051X.1988.tb01584.x

5. Cobb CM. Non-surgical pocket therapy: mechanical. Ann Periodontol. 1996 Nov; (1):443-90. https://doi.org/10.1902/annals.1996.1.1.443

6. Shanbhag S, Dahiya M, Croucher R. The impact of periodontal therapy on oral health-related quality of life in adults: a systematic review. J Clin Periodontol. 2012 Aug;39(8):725-35. https://doi.org/10.1111/j.1600-051X.2012.01910.x

7. Hirschfeld L, Wasserman B. A long-term survey of tooth loss in 600 treated periodontal patients. J Periodontol. 1978 May;49(5):225-37. https://doi.org/10.1902/jop.1978.49.5.225 disease and may also equip us with specific indicators for individualized periodontal treatment (Personalized/ Precision Medicine) that will lead to more predictable and effective treatment outcome for all patients with less frequent need for periodontal surgery or tooth loss than we have today.

\section{Conclusions}

This prospective cohort study found that IL-10 rs1800872 polymorphism was associated with an increased risk of further disease progression and the potential need for further treatment following non-surgical periodontal treatment. On the other hand, susceptible IL-6 genotypes were not associated with the risk of persisting or recurrent disease activity.

8. McGuire MK, Nunn ME. Prognosis versus actual outcome. III. The effectiveness of clinical parameters in accurately predicting tooth survival. J Periodontol. 1996 Jul;67(7):666-74. https://doi.org/10.1902/jop.1996.67.7.666

9. Chambrone LA, Chambrone L. Tooth loss in well-maintained patients with chronic periodontitis during long-term supportive therapy in Brazil. J Clin Periodontol. 2006 Oct;33(10):759-64 https://doi.org/10.1111/j.1600-051X.2006.00972.x

10. Faggion CM Jr, Petersilka G, Lange DE, Gerss J, Flemmig TF. Prognostic model for tooth survival in patients treated for periodontitis. J Clin Periodontol. 2007 Mar;34(3):226-31. https://doi.org/10.1111/i.1600-051X.2006.01045.x

11. Matuliene G, Pjetursson BE, Salvi GE, Schmidlin K, Brägger U, Zwahlen $\mathrm{M}$ et al. Influence of residual pockets on progression of periodontitis and tooth loss: results after 11 years of maintenance. J Clin Periodontol. 2008 Aug;35(8):685-95. https://doi.org/10.1111/i.1600-051X.2008.01245.x

12. Pihlstrom BL, Michalowicz BS, Johnson NW. Periodontal diseases. Lancet. 2005 Nov;366(9499):1809-20. https://doi.org/10.1016/S0140-6736(05)67728-8

13. Giannobile WV, Beikler T, Kinney JS, Ramseier CA, Morelli T, Wong DT. Saliva as a diagnostic tool for periodontal disease: current state and future directions. Periodontol 2000. 2009;50(1):52-64. https://doi.org/10.1111/i.1600-0757.2008.00288.x

14. Yoshie H, Kobayashi T, Tai H, Galicia JC. The role of genetic polymorphisms in periodontitis. Periodontol 2000. 2007;43(1):102-32. https://doi.org/10.1111/j.1600-0757.2006.00164.x 
15. Chatzopoulos GS, Doufexi AE, Kalogirou F. Association of susceptible genotypes to periodontal disease with the clinical outcome and tooth survival after non-surgical periodontal therapy: A systematic review and meta-analysis. Med Oral Patol Oral Cir Bucal. 2016 Jan;21(1):e14-29. https://doi.org/10.4317/medoral.20638 PMID:26595831

16. Ridker PM, Rifai N, Stampfer MJ, Hennekens CH. Plasma concentration of interleukin- 6 and the risk of future myocardial infarction among apparently healthy men. Circulation. 2000 Apr;101(15):1767-72. https://doi.org/10.1161/01.CIR.101.15.1767

17. McCauley LK, Nohutcu RM. Mediators of periodontal osseous destruction and remodeling: principles and implications for diagnosis and therapy. J Periodontol. 2002 Nov;73(11):1377-91. https://doi.org/10.1902/jop.2002.73.11.1377

18. Moreira PR, Lima PM, Sathler KO, Imanishi SA, Costa JE, Gomes RS et al. Interleukin-6 expression and gene polymorphism are associated with severity of periodontal disease in a sample of Brazilian individuals. Clin Exp Immunol. 2007 Apr;148(1):119-26. https://doi.org/10.1111/j.1365-2249.2007.03327.x

19. Hughes FJ, Turner W, Belibasakis G, Martuscelli G. Effects of growth factors and cytokines on osteoblast differentiation. Periodontol 2000 2006;41.48-72. https://doi.org/10.1111/j.1600-0757.2006.00161.x.

20. Hulkkonen J, Pertovaara M, Antonen J, Pasternack A, Hurme M. Elevated interleukin- 6 plasma levels are regulated by the promoter region polymorphism of the IL6 gene in primary Sïgren's syndrome and correlate with the clinical manifestations of the disease. Rheumatology (Oxford). 2001 Jun;40(6):656-61. https://doi.org/10.1093/rheumatology/40.6.656PMID:11426023

21. Morse HR, Olomolaiye OO, Wood NA, Keen LJ, Bidwell JL. Induced heteroduplex genotyping of TNF-alpha, IL-1beta, IL- 6 and IL-10 polymorphisms associated with transcriptional regulation. Cytokine. 1999 Oct;11(10):789-95. https://doi.org/10.1006/cyto.1999.0491 PMID:10525318

22. Groux H, O'Garra A, Bigler M, Rouleau M, Antonenko S, Vries JE et al. A CD4+ T-cell subset inhibits antigen-specific T-cell responses and prevents colitis. Nature. 1997 Oct;389(6652):73742. https://doi.org/10.1038/39614 PMID:9338786

23. Albuquerque CM, Cortinhas AJ, Morinha FJ, Leitão JC, Viegas CA, Bastos EM. Association of the IL-10 polymorphisms and periodontitis: a meta-analysis. Mol Biol Rep. 2012 Oct;39(10):9319-29. https://doi.org/10.1007/s11033-012-1738-1

24. Song GG, Choi SJ, Ji JD, Lee YH. Association between tumor necrosis factor- $\alpha$ promoter $-308 \mathrm{~A} / \mathrm{G},-238 \mathrm{~A} / \mathrm{G}$, interleukin-6 - 174 G/C and -572 G/C polymorphisms and periodontal disease: a meta-analysis. Mol Biol Rep. 2013 Aug;40(8):5191-203. https://doi.org/10.1007/s11033-013-2621-4PMID:23657600
25. Armitage GC. Development of a classification system for periodontal diseases and conditions. Ann Periodontol. 1999 Dec;4(1):1-6. https://doi.org/10.1902/annals.1999.4.1.1

26. Tonetti MS, Claffey N. Advances in the progression of periodontitis and proposal of definitions of a periodontitis case and disease progression for use in risk factor research. Group C consensus report of the 5th European Workshop in Periodontology. J Clin Periodontol. 2005;32(s6 Suppl 6):210-3. https://doi.org/10.1111/j.1600-051X.2005.00822.x

27. Chatzopoulos GS, Doufexi AE, Kouvatsi A. Clinical response to non-surgical periodontal treatment in patients with interleukin-6 and interleukin-10 polymorphisms. Med Oral Patol Oral Cir Bucal. 2017 Jul;22(4):e446-57. https://doi.org/10.4317/medoral.21795

28. Kaldahl WB, Kalkwarf KL, Patil KD, Molvar MP, Dyer JK. Long-term evaluation of periodontal therapy: II. Incidence of sites breaking down. J Periodontol. 1996 Feb;67(2):103-8. https://doi.org/10.1902/jop.1996.67.2.103

29. Schäfer AS, Jepsen S, Loos BG. Periodontal genetics: a decade of genetic association studies mandates better study designs. J Clin Periodontol. 2011 Feb;38(2):103-7. https://doi.org/10.1111/j.1600-051X.2010.01653.x

30. Lamster IB, Hartley LJ, Vogel RI. Development of a biochemical profile for gingival crevicular fluid. Methodological considerations and evaluation of collagen-degrading and ground substance-degrading enzyme activity during experimental gingivitis. J Periodontol. 1985 Nov;56(11 Suppl):13-21. https://doi.org/10.1902/jop.1985.56.11s.13

31. Fonseca JE, Santos MJ, Canhão H, Choy E. Interleukin-6 as a key player in systemic inflammation and joint destruction. Autoimmun Rev. 2009 Jun;8(7):538-42. https://doi.org/10.1016/j.autrev.2009.01.012

32. Schätzle M, Löe $H$, Bürgin W, Anerud A, Boysen H, Lang NP. Clinical course of chronic periodontitis. I. Role of gingivitis. J Clin Periodontol. 2003 Oct;30(10):887-901. https://doi.org/10.1034/j.1600-051X.2003.00414.x

33. Joss A, Adler R, Lang NP. Bleeding on probing. A parameter for monitoring periodontal conditions in clinical practice. J Clin Periodontol. 1994 Jul;21(6):402-8. https://doi.org/10.1111/j.1600-051X.1994.tb00737.x

34. Lang NP, Joss A, Orsanic T, Gusberti FA, Siegrist BE. Bleeding on probing. A predictor for the progression of periodontal disease? J Clin Periodontol. 1986 Jul;13(6):590-6. https://doi.org/10.1111/j.1600-051X.1986.tb00852.x 